\title{
Thermal Stability of the Plasma Membrane Calcium Pump. Quantitative Analysis of Its Dependence on Lipid-Protein Interactions
}

\author{
V. Levi, J.P.F.C. Rossi, M.M. Echarte, P.R. Castello, F.L. González Flecha \\ Instituto de Química y Fisicoquímica Biológicas_-Departamento de Química Biológica, Facultad de Farmacia y Bioquímica, Universidad de \\ Buenos Aires, Junín 956-1113 Buenos Aires, Argentina
}

Received: 5 August 1999/Revised: 20 October 1999

\begin{abstract}
Thermal stability of plasma membrane $\mathrm{Ca}^{2+}$ pump was systematically studied in three micellar systems of different composition, and related with the interactions amphiphile-protein measured by fluorescence resonance energy transfer. Thermal denaturation was characterized as an irreversible process that is well described by a first order kinetic with an activation energy of $222 \pm 12 \mathrm{~kJ} / \mathrm{mol}$ in the range $33-45^{\circ} \mathrm{C}$. Upon increasing the mole fraction of phospholipid in the mixed micelles where the $\mathrm{Ca}^{2+}$ pump was reconstituted, the kinetic coefficient for the inactivation process diminished until it reached a constant value, different for each phospholipid species. We propose a model in which thermal stability of the pump depends on the composition of the amphiphile monolayer directly in contact with the transmembrane protein surface. Application of this model shows that the maximal pump stability is attained when $80 \%$ of this surface is covered by phospholipids. This analysis provides an indirect measure of the relative affinity phospholipid/detergent for the hydrophobic transmembrane surface of the protein $\left(K_{L D}\right)$ showing that those phospholipids with higher affinity provide greater stability to the $\mathrm{Ca}^{2+}$ pump. We developed a method for directly measure $K_{L D}$ by using fluorescence resonance energy transfer from the membrane protein tryptophan residues to a pyrene-labeled phospholipid. $K_{L D}$ values obtained by this procedure agree with those obtained from the model, providing a strong evidence to support its validity.
\end{abstract}

Key words: Membrane proteins $-\mathrm{Ca}^{2+}$-ATPase PMCA - Thermal inactivation - Micellar phase Protein-amphiphiles interactions

\section{Introduction}

The erythrocyte $\mathrm{Ca}^{2+}$ pump is a single chain integral membrane protein that actively transports $\mathrm{Ca}^{2+}$ from the cytoplasm to the external milieu. A large portion of the pump faces the cytoplasm, and short external loops connect 8 to 10 putative transmembrane segments (Møller, Juul \& le Maire, 1996). Despite several years of research aimed at unraveling the functional and structural properties of the $\mathrm{Ca}^{2+}$ pump, essential aspects of its structure and catalytic mechanism remain unsolved.

Denaturation of proteins implies the disruption of their native, three-dimensional conformation (Creighton, 1993). The forces involved in the folding and unfolding of proteins, which are closely related to the properties of the surrounding medium determine their stability (Rees et al., 1989; Popot \& Engelman, 1990). Thus, denaturation occurs as a consequence of environmental alterations such as changes in temperature or pressure, or the presence of certain chemical agents. While thermal denaturation of globular proteins is a well-studied process, relatively little is known about the thermal stability of membrane proteins (Creighton, 1993; Lau \& Bowie, 1997).

Most of the studies on membrane proteins require the use of amphiphiles for protein isolation, reconstitution and crystallization (Ostermeier \& Michel, 1997). The employment of these amphiphiles involve the disruption of the membrane and the protein inclusion in an artificial medium, i.e., micelles, in which the amphiphiles constitute the solvent of the hydrophobic transmembrane domain of the protein. Therefore, the study of the interactions between membrane proteins and amphiphiles and the dependence of the enzyme properties on these interactions, constitute important topics for the determination of the optimal conditions for protein reconstitution. 
Our purpose in this work is to precisely describe the process of thermal denaturation of the purified plasma membrane $\mathrm{Ca}^{2+}$-ATPase and its dependence on the micelle composition. We perform a kinetic analysis and develop a fluorescence-spectroscopic method for describing the interactions amphiphile-protein, and the effects of these interactions on protein stability.

\section{Materials and Methods}

\section{MATERIALS}

All the chemicals used in this work were of analytical grade and mostly purchased from Sigma Chemical (St. Louis, MO). SM-2 Bio-Beads were obtained from Bio-Rad Laboratories (Hercules, CA). 3-(trifluoromethyl)-3-( $m$ - $\left[{ }^{125} \mathrm{I}\right]$ iodophenyl $)$-diazirine, $\left[{ }^{125} \mathrm{I}\right] \mathrm{TID}(2.8 \mathrm{mCi} / \mathrm{ml}$ in methanol:water 3:1 v/v) was brought from Amersham International plc (UK). $\left[{ }^{125} \mathrm{I}\right] \mathrm{TID}$ labeled $\mathrm{C}_{12} \mathrm{E}_{10}$ was prepared according to the procedure described by Castello et al. (1997) with some modifications (Levi et al., personal communication). Soybean phospholipids (type II-S) were purchased from Sigma Chemical (St. Louis, MO). HPPC was obtained from Molecular Probes (Eugene, OR) and store in a stock solution (1.18 mM in chloroform). Recently drawn human blood was obtained from the Hematology Section of the Hospital de Clinicas General José de San Martín (Argentina).

\section{PURIFICATION OF $\mathrm{Ca}^{2+}$ PUMP FROM HUMAN ERYTHROCYTES}

Calmodulin-depleted erythrocyte membranes were prepared as described previously (González-Flecha et al., 1999) using 15 mM MOPS (pH 7.4 at $4^{\circ} \mathrm{C}$ ), 0.1 mM PMSF and 1 mM EGTA as hypotonic solution. $\mathrm{Ca}^{2+}$-ATPase was isolated in pure form by calmodulin affinity chromatography (Castello et al., 1994). Fractions exhibiting the highest specific ATPase activity were pooled. Homogeneity was verified by SDS-PAGE (single band at $M_{r} 134,000$ ) and the $\mathrm{Ca}^{2+}$ pump concentration was measured by densitometric analysis. Phospholipid concentration was determined according to Chen, Toribara and Warner (1956). Prior to use, the enzyme $(78 \mu \mathrm{g} / \mathrm{ml}$, specific ATPase activity $11.5 \mu \mathrm{mol} \mathrm{Pi} / \mathrm{mg} \mathrm{min}$ ) was kept, under liquid nitrogen, in storage buffer $\left(300 \mathrm{~mm} \mathrm{KCl}, 10 \mathrm{~mm}\right.$ MOPS-K (pH 7.4 at $\left.4^{\circ} \mathrm{C}\right), 1 \mathrm{~mm} \mathrm{MgCl}_{2}, 2 \mathrm{~mm}$ EDTA, $2 \mathrm{~mm} \mathrm{CaCl}_{2}\left(\left[\mathrm{Ca}^{2+}\right]_{\text {free }}=68.7 \mu \mathrm{M}\right)$ and $2 \mathrm{~mm}$ DTT$)$ with 290 $\mu \mathrm{M}$ soybean phospholipid and $800 \mu \mathrm{M} \mathrm{C}_{12} \mathrm{E}_{10}$ except where indicated. The critical micellar concentration of $\mathrm{C}_{12} \mathrm{E}_{10}$ in the storage buffer, was measured according to Casey and Reinhart (1993) considering that the mean $M_{r}$ of $\mathrm{C}_{12} \mathrm{E}_{10}$ is 627 (Ganong \& Lu, 1989).

\section{ISOLATION OF MEMBRANE PHOSPHOLIPIDS}

Erythrocyte lipids were extracted using the method of Wang et al. (1994). Phospholipid concentrations were measured according to Chen et al. (1956).

\section{DETERGENT REMOval}

To remove the detergent from the solubilized $\mathrm{Ca}^{2+}$ pump, $0.6 \mathrm{~g} / \mathrm{ml}$ prewashed wet Bio-Beads SM-2 absorbent (20-50 mesh) were added, and the mixture was incubated for $1.5 \mathrm{hr}$ at room temperature with continuous stirring. Finally, the Bio-Beads were separated by centrifu- gation for $3 \mathrm{~min}$ at $1,000 \times g$. Detergent removal was monitored in a parallel experiment using $\left[{ }^{125} \mathrm{I}\right] \mathrm{TID}$ labeled $\mathrm{C}_{12} \mathrm{E}_{10}$.

\section{MeAsurement of the $\mathrm{Ca}^{2+}$-ATPase Activity}

ATPase activity was measured at $37^{\circ} \mathrm{C}$ as the initial velocity of release of Pi from ATP, as described previously (González Flecha et al., 1999), with some modifications. The incubation medium was: $120 \mathrm{mM} \mathrm{KCl}$; 30 mм MOPS-K (pH 7.4); $3.75 \mathrm{~mm} \mathrm{MgCl}_{2} ; 1$ mM EGTA; $1.1 \mathrm{~mm}$ $\mathrm{CaCl}_{2}\left(\left[\mathrm{Ca}^{2+}\right]_{\text {free }}=140 \mu \mathrm{M}\right) ; 140 \mu \mathrm{M}$ soybean phospholipids; $800 \mu \mathrm{M}$ $\mathrm{C}_{12} \mathrm{E}_{10}$ and $2 \mathrm{~mm}$ ATP. The protein concentration was $1 \mu \mathrm{g} / \mathrm{ml}$. $\mathrm{Ca}^{2+}$ ATPase activity was measured as the difference between the activity in the aforementioned medium and that measured in the same medium without $\mathrm{Ca}^{2+}$. Release of $\mathrm{Pi}$ was estimated according to the procedure of Fiske and Subbarow (1925). The concentration of $\mathrm{Ca}^{2+}$ in the incubation medium was measured using an Orion 9320 ion-selective $\mathrm{Ca}^{2+}$ electrode (Beverly, MA).

\section{THERMAL INACTIVATION}

Thermal inactivation experiments were performed by incubating the $\mathrm{Ca}^{2+}$ pump $(10 \mu \mathrm{g})$ in the storage buffer containing the phospholipid and detergent concentrations detailed in each case. To prevent proteolysis and bacterial growth $3 \mathrm{~mm}$ sodium azide; $1 \mu \mathrm{M}$ pepstatine; $10 \mu \mathrm{M}$ leupeptin; $1 \mu \mathrm{g} / \mathrm{ml}$ aprotinin and $0.1 \mathrm{~mm}$ PMSF were added to the medium. The tubes were sealed and covered to avoid evaporation and to protect the system from light.

\section{Polyacrylamide Gel EleCtrophoresis}

Prior to electrophoresis, samples $(0.5 \mu \mathrm{g}$ per lane), were incubated at room temperature for 5-10 $\mathrm{min}$ in sample buffer. SDS-PAGE (10\% T and $1 \% \mathrm{C}$ ) was carried out according to the Tris/tricine method (Schägger \& von Jagow, 1987). Gels were then stained with colloidal Brilliant Blue G (Neuhoff et al., 1988).

\section{Fluorescence Measurements}

$\mathrm{Ca}^{2+}$ pump emission spectrum was measured at $25^{\circ} \mathrm{C}$ in a $3 \times 3 \mathrm{~mm}$ quartz cuvette using a SLM-AMINCO BOWMAN Series 2 spectrofluorometer (Spectronic Instrument, Rochester, NY) with excitation at $290 \mathrm{~nm}$. Both excitation and emission bandwidths were set at $4 \mathrm{~nm}$. Each spectrum was measured between 300-400 nm and was corrected for background emission.

Fluorescence energy transfer was measured from the decrease on $\mathrm{Ca}^{2+}$-ATPase intrinsic fluorescence caused by the presence of the acceptor HPPC. According to Förster (1948), $R_{o}$ (the distance in $\AA$ at which the transfer efficiency is $50 \%$ for a single donor-acceptor pair) is given by:

$R_{o}=9.79 \times 10^{3} \cdot\left(\frac{\kappa^{2} \cdot \phi_{D} \cdot J}{\eta^{4}}\right)^{1 / 6}$

where $\eta$ is the refractive index of the medium, $\kappa^{2}$ is the orientation factor between the transition moments of the donor and acceptor, $\phi_{D}$ is the fluorescence quantum yield of the energy donor in the absence of acceptor and $J$ is the spectral overlap integral $\left(\right.$ in $\mathrm{cm}^{3} \mathrm{M}^{-1}$ ) defined by:

$J=\int I_{D}(\lambda) \cdot \varepsilon_{A}(\lambda) \cdot \lambda^{4} \cdot d \lambda$ 
in which $\lambda$ is the wavelength, $I_{D}(\lambda)$ is the fluorescence of the donor with the total intensity normalized to unity, and $\varepsilon_{A}(\lambda)$ is the extinction coefficient of the acceptor, both at $\lambda$.

\section{Measurement of the Displacement Constant of $\mathrm{C}_{12} \mathrm{E}_{10}$ By Phospholipids on the Transmembrane SURFACE OF THE $\mathrm{Ca}^{2+}$ PUMP BY FRET}

The relative affinity of the different phospholipidic species respect to $\mathrm{C}_{12} \mathrm{E}_{10}$ for the hydrophobic transmembrane surface of the $\mathrm{Ca}^{2+}$-ATPase was studied by measuring the decrease on the fluorescence emission of the protein due to the addition of a fluorescence acceptor labeled phospholipid (HPPC)

Similarly as described by Veatch and Stryer (1977), the decrease on the $\mathrm{Ca}^{2+}$ pump emission could be directly related to the fraction of the hydrophobic transmembrane surface that is cover by HPPC according to:

$\frac{I_{d, a}}{I_{d}}=1-E \cdot \theta_{H P P C}$

where $I_{d, a}$ and $I_{d}$ are the fluorescence intensities of the pump in the presence and the absence of the acceptor respectively, $E$ is the FRET efficiency and $\theta_{H P P C}$ is the fractional coverage of the hydrophobic transmembrane surface of the protein with HPPC. By replacing $\theta_{H P P C}$ with the corresponding expressions derived from the adsorption isotherm of amphiphiles and considering that most of the contact sites are occupied by unlabeled amphiphiles, the following relation is obtained:

$\frac{I_{d, a}}{I_{d}} \cong 1-X_{H P P C, m i c} \cdot \frac{\xi}{K_{L D} \cdot X_{L, m i c}+X_{D, m i c}{ }^{\beta}}$

where $\xi$ is a constant for each system, $\beta$ and $K_{L D}$ are the stoichiometric coefficient and the displacement constant for the exchange $\mathrm{C}_{12} \mathrm{E}_{10}: \mathrm{PL}$ on the transmembrane surface of the $\mathrm{Ca}^{2+}$ pump, respectively (see Appendix for details).

\section{DATA ANALYSIS}

All measurements were performed in duplicate or triplicate. Data presented in Results included at least two independent experiments. The equations were fitted to the experimental data using a multivariate nonlinear regression procedure based on the Gauss-Newton algorithm (Seber \& Wild, 1989). The dependent variable was assumed homoscedastic (constant variance), and the independent variable was considered to have negligible error. The "equation of best fit" was deemed that which gave the minimal Akaike index (Akaike, 1974) and the least biased fit. Parameters were expressed as the mean \pm SE.

$\begin{array}{ll}\text { ABBREVIATIONS } \\ \text { BHT } & \text { butylated hydroxytoluene } \\ \mathrm{C}_{12} \mathrm{E}_{10} & \text { poly(oxyethylene)10-lauryl ether } \\ \text { DOPC } & \text { 1,2-dioleoyl-sn-glycero-3-phosphocholine } \\ \text { DTT } & \text { 1,4-dithiothreitol } \\ \text { FRET } & \text { fluorescence resonance energy transfer } \\ \text { HPPC } & \text { 1-hexadecanoyl-2-(1 pyrenedecanoyl)-sn-glycero-3-phos- } \\ & \text { phocholine } \\ K_{L D} & \begin{array}{l}\text { displacement constant for the exchange } \mathrm{C}_{12} \mathrm{E}_{10} \text { phospholipid } \\ \text { on the transmembrane surface of the membrane protein }\end{array} \\ \text { PL } & \begin{array}{l}\text { phospholipid } \\ X_{i, m i c}\end{array}\end{array}$

\section{Results}

\section{Kinetics of $\mathrm{Ca}^{2+}$-ATPAse Thermal Denaturation}

Thermal inactivation of solubilized $\mathrm{Ca}^{2+}$ pump was studied by incubating at different temperatures the purified enzyme in storage buffer with $290 \mu \mathrm{M}$ soybean phospholipids and $800 \mu \mathrm{M} \mathrm{C}_{12} \mathrm{E}_{10}$. After different incubation periods at each temperature, $\mathrm{Ca}^{2+}$-ATPase activity $(v)$ was measured as described in Materials and Methods.

Figure $1 A$ shows the time course of the inactivation of the enzyme within a temperature range $33-45^{\circ} \mathrm{C}$. The fact that the $\mathrm{Ca}^{2+}$-ATPase activity measured at $37^{\circ} \mathrm{C}$ is not restored after the incubation indicates that thermal inactivation of the enzyme is an irreversible process. The best fit to the data was obtained by applying Eq. 5:

$\frac{v}{v_{o}}=e^{-k \cdot t}$

where $k$ is the inactivation rate coefficient, $v$ is the remaining activity, $v_{o}$ is the initial activity and $t$ is the incubation time.

The relation between $k$ and temperature is described by the Arrhenius equation for many reactions:

$\ln \left(\frac{k}{k_{0}}\right)=\ln A-\frac{E a}{R \cdot T}$

where $A$ is the pre-exponential factor, $E a$ is the activation energy for thermal inactivation, $R$ is the gas constant, $T$ is the absolute temperature and $k_{0}$ is the reference rate coefficient $\left(k_{0} \equiv 1 \mathrm{hr}^{-1}\right)$. Figure $1 B$ shows the values of $\ln \left(k / k_{0}\right)$ plotted as a function of $T^{-1}$. By fitting Eq. 6 to the previously found values of $k, E a=222 \pm 12 \mathrm{~kJ} / \mathrm{mol}$ and $\ln A=83 \pm 5$ were obtained. The linearity of $\mathrm{Ar}$ rhenius plot suggests that a single common mechanism is responsible for $\mathrm{Ca}^{2+}$ pump inactivation over the range of temperatures assayed. Therefore, as a practical matter, further inactivation experiments were run at $44^{\circ} \mathrm{C}$ so they could be completed within $3 \mathrm{hr}$.

Both native and thermally inactivated $\mathrm{Ca}^{2+}$ pump, migrated as a single band of $M_{r}, 134,000$ after SDSPAGE (data not shown), indicating that thermal inactivation was not due to either fragmentation or formation of SDS-stable aggregates of the protein. To determine whether the inactivation of the enzyme was related to the presence of residual amounts of peroxides in the detergent (Møller, le Maire \& Andersen, 1986), various concentrations of the free radical scavenger BHT (0 to $4 \mu \mathrm{M})$ were added to purified preparations of $\mathrm{Ca}^{2+}$-ATPase. The samples were then incubated for selected periods at $44^{\circ} \mathrm{C}$ under the conditions indicated above; $\mathrm{Ca}^{2+}$-ATPase activity was measured and found to be independent of the BHT concentration. 

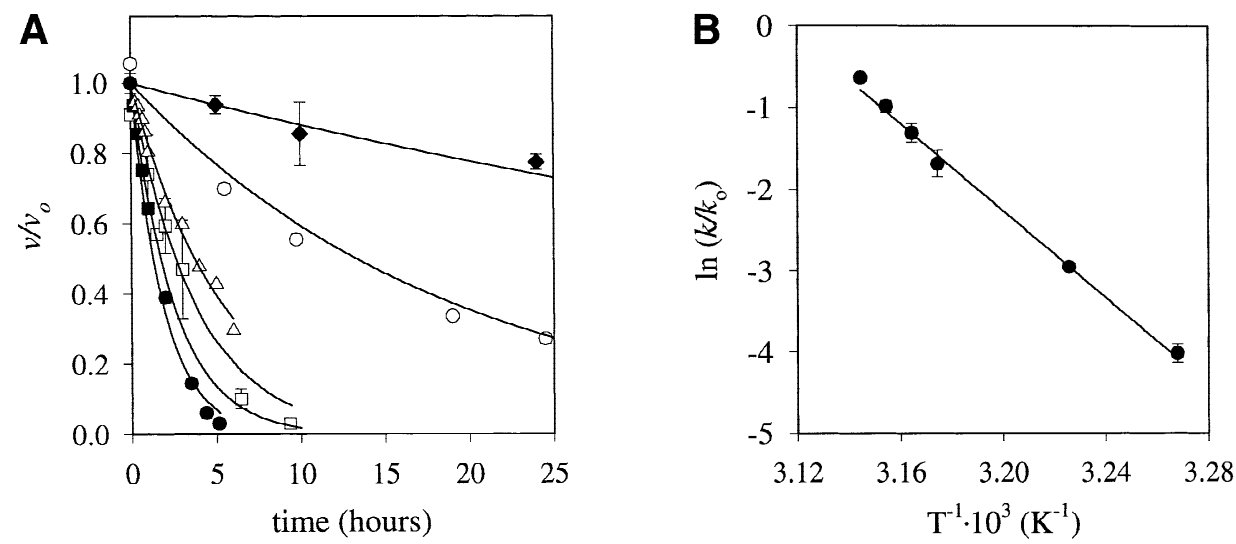

Fig. 1. Kinetics of thermal inactivation of the $\mathrm{Ca}^{2+}$-ATPase. (A) Time course of the thermal inactivation of the Ca ${ }^{2+}$ pump. The enzyme was incubated in storage buffer with $290 \mu \mathrm{M}$ soybean phospholipids and $800 \mu \mathrm{M} \mathrm{C}_{12} \mathrm{E}_{10}$ under the conditions described in Materials and Methods. Incubation temperatures were: $33^{\circ} \mathrm{C}(\diamond), 37^{\circ} \mathrm{C}(\bigcirc), 42^{\circ} \mathrm{C}(\triangle), 43^{\circ} \mathrm{C}(\square), 44^{\circ} \mathrm{C}(\boldsymbol{\square})$ and $45^{\circ} \mathrm{C}(\bullet)$. At selected periods of incubation, aliquots were taken, and $\mathrm{Ca}^{2+}$-ATPase activity was assayed. Relative activity was plotted as a function of the incubation time. The continuous lines correspond to the fitting of Eq. 5 to the experimental data. The best-fitting values of $k$ were $0.018 \pm 0.002 \mathrm{hr}^{-1}\left(33^{\circ} \mathrm{C}\right) ; 0.052 \pm 0.002 \mathrm{hr}^{-1}\left(37^{\circ} \mathrm{C}\right) ; 0.18 \pm 0.03$ $\mathrm{hr}^{-1}\left(42^{\circ} \mathrm{C}\right), 0.27 \pm 0.03 \mathrm{hr}^{-1}\left(43^{\circ} \mathrm{C}\right) ; 0.40 \pm 0.03 \mathrm{hr}^{-1}\left(44^{\circ} \mathrm{C}\right)$ and $0.53 \pm 0.02 \mathrm{hr}^{-1}\left(45^{\circ} \mathrm{C}\right)$. (B) Temperature dependence of the kinetic coefficients of thermal inactivation of $\mathrm{Ca}^{2+}$-ATPase. The straight line is the graphical representation of Eq. 6 obtained using the best-fit parameter values indicated in the text.

\section{Factors AfFecting Thermal Denaturation of the Solubilized $\mathrm{Ca}^{2+}$ Pump}

The following experiments were designed to evaluate the effect of modifying micelle composition on the thermal stability of the purified $\mathrm{Ca}^{2+}$ pump.

Throughout this work, micelles were considered as a separate phase (Tanford, 1980) whose composition is defined by the mole fractions of its components:

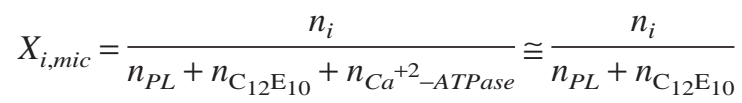

where $X_{i, m i c}$ is the mole fraction of the $i$ component in the micelles; $i$ may be phospholipid, detergent or protein; and $n$ refers to the total amount of substance (number of moles). In all assays $n_{\mathrm{Ca}^{2+} \text {-ATPase }}<<n_{P L}$, meaning that the approximation in Eq. 7 is adequate. According to the low critical micellar concentrations of phospholipids (Tanford, 1980) and $\mathrm{C}_{12} \mathrm{E}_{10}(5.1 \mu \mathrm{M})$ we presumed that both amphiphiles were present in the micellar phase exclusively.

\section{Effect of Phospholipid Mole Fraction}

Initially, the $\mathrm{Ca}^{2+}$ pump was purified and reconstituted in storage buffer with $14 \mu \mathrm{M}$ soybean phospholipids and $800 \mu \mathrm{M} \mathrm{C}_{12} \mathrm{E}_{10}$. This preparation was then supplemented with soybean phospholipids up to the concentrations indicated in the legend to Fig. $2 A$, while the concentrations of the other components were kept constant.
The inset to the figure shows the time course of thermal inactivation at $44^{\circ} \mathrm{C}$. Equation 5 was fitted to the experimental data, and the rate coefficients were estimated and plotted as a function of the soybean phospholipids mole fraction. This figure shows that the stability of the $\mathrm{Ca}^{2+}$ pump increased with $X_{\text {soybean } P L, \text { mic }}$ up to 0.15 and $k$ became constant beyond this value.

The dependence of $k$ on the phospholipid mole fraction was further studied by repeating the same procedure except that the $\mathrm{Ca}^{2+}$ pump was purified in the presence of either erythrocyte membrane lipids (Fig. 2B) or DOPC (Fig. 2C) instead of soybean phospholipids. In these cases, the dependence of $k$ on the mole fraction of each phospholipid was similar to that observed with soybean phospholipids: $k$ was steeply dependent on phospholipid mole fraction, but beyond a certain value $(>0.5$ for $X_{\text {erythrocyte } P L, \text { mic }}$ and $>0.6$ for $\left.X_{D O P C, m i c}\right)$, this dependence was minimal. Comparison of Fig. $2 A, B$ and $C$ reveals that the maximal stability of the calcium pump increased according to the following sequence: soybean PL > erythrocyte PL >> DOPC.

\section{Effect of the Detergent Mole Fraction}

$\mathrm{C}_{12} \mathrm{E}_{10}$ concentration in preparations of purified $\mathrm{Ca}^{2+}$ pump was adjusted to the values indicated in the legend to Fig. 3. Samples were incubated at $44^{\circ} \mathrm{C}$, and $\mathrm{Ca}^{2+}$ ATPase activity was measured as a function of time. Equation 5 was fitted to the experimental data, and the rate coefficients for the thermal inactivation were estimated and plotted as a function of the detergent mole fraction (Fig. 3). 

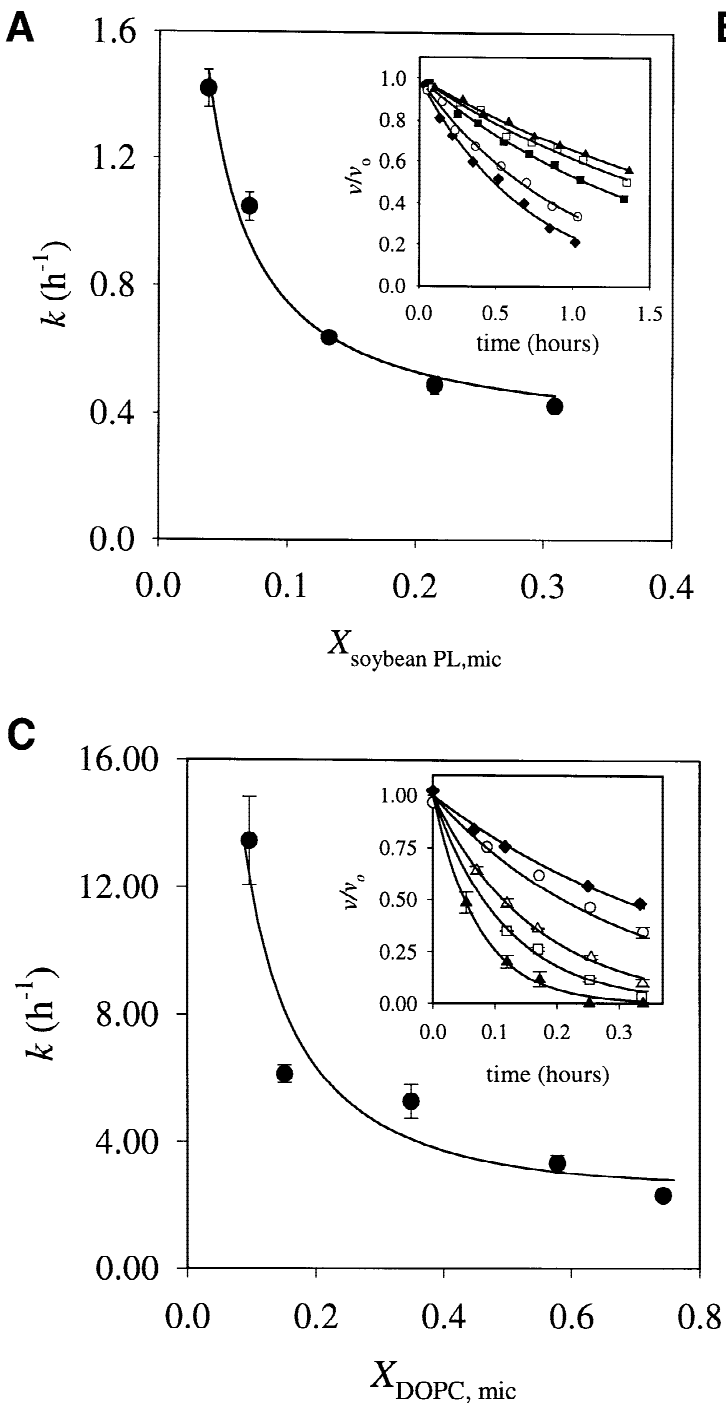

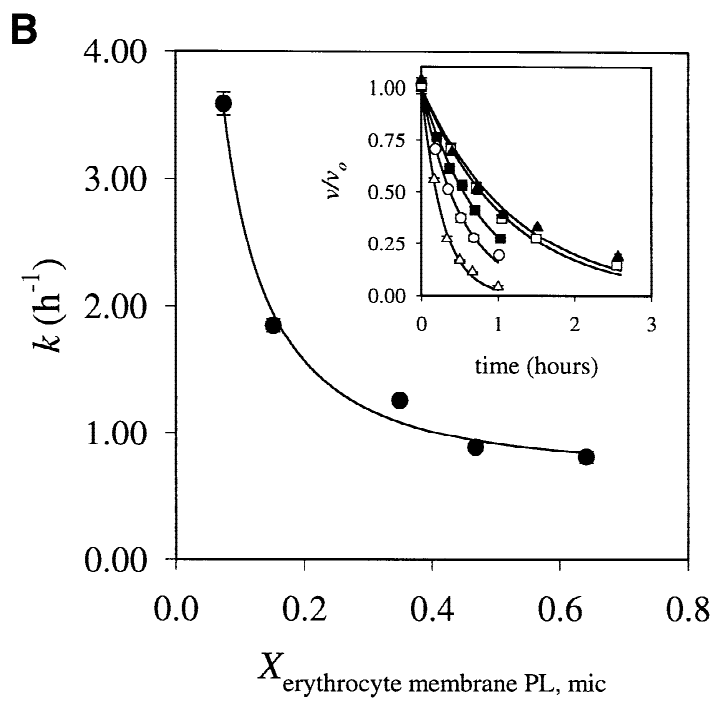

Fig. 2. Dependence of the kinetic coefficient for the thermal inactivation on phospholipid mole fraction. $\mathrm{Ca}^{2+}$-ATPase was purified and reconstituted in storage buffer in the presence of $800 \mu \mathrm{M} \mathrm{C}{ }_{12} \mathrm{E}_{10}$ and soybean phospholipids $(A)$, phospholipids extracted from erythrocyte membranes $(B)$ or DOPC $(C)$. The phospholipid concentrations were $(\mu \mathrm{M})$ : soybean phospholipids: $32(\diamond), 60(\bigcirc), 121(\boldsymbol{\square}), 217(\square)$ and $362(\mathbf{\Delta})$; erythrocyte membrane phospholipids: $641(\triangle), 140(\bigcirc), 440$ $(\mathbf{\square}), 710(\square)$ and $1430(\mathbf{\Delta})$ and; DOPC: $86(\mathbf{\Delta}), 140(\square), 430(\triangle)$, $1100(\bigcirc)$ and $2300(\diamond)$. Samples were incubated at $44^{\circ} \mathrm{C}^{-\mathrm{Ca}^{2+} \text {-ATPase }}$ activity was measured as described in Materials and Methods and plotted as a function of the incubation time (insets). Values of $k$ were obtained by fitting Eq. 5 to the experimental data and represented as a function of $X_{L, m i c}$. The continuous lines are the graphical representatioin of Eq. 10 with the following best fitting parameters:

\section{Soybean PL}

$\begin{array}{ccc}K_{L D} & k_{1}\left(\mathrm{hr}^{-1}\right) & \beta \\ 9 \pm 2 & 0.39 \pm 0.05 & 2 \\ 3.3 \pm 0.4 & 0.80 \pm 0.07 & 2 \\ 2 \pm 1 & 2.6 \pm 0.9 & 2\end{array}$

This figure shows that $k$ for the thermal inactivation of the $\mathrm{Ca}^{2+}$ pump was independent of the $\mathrm{C}_{12} \mathrm{E}_{10}$ mole fraction in the range $0.4-0.85$; beyond this range, $k$ increased steeply.

\section{Resonance Energy Transfer Measurements}

To determine $K_{L D}$, we used steady state fluorescence resonance energy transfer between natural fluorophores of the $\mathrm{Ca}^{2+}$-ATPase and a fluorescent labeled phospholipid. In these experiments, Trp residues of the $\mathrm{Ca}^{2+}$ pump constituted the donors and pyrene bound to the phosphatidylcholine acted as the energy acceptor. The donor-acceptor pair used in this system has the advantage of the high extinction coefficient of the acceptor and an excellent degree of overlap between the emission of the protein and the absorption spectrum of HPPC (Fig. $4 A$ ). The overlap integral $J$, calculated as described in
Materials and Methods, was $1.7 \times 10^{14} \mathrm{~cm}^{3} \mathrm{M}^{-1}$. The quantum yield of Trp residues in the $\mathrm{Ca}^{2+}$-ATPase was found to be 0.03 , using $\operatorname{Trp}$ in water $\left(25^{\circ} \mathrm{C}\right)$ as a standard (Wu \& Brand, 1994). Taking into account these values and considering 1.33 for the refractive index and $2 / 3$ for the orientation factor $\kappa^{2}$ (Lakowicz, 1983), the calculated Förster distance $R_{o}$ was $21.4 \AA$.

Figure $4 B$ shows the emission spectrum of the $\mathrm{Ca}^{2+}$ pump in the absence of acceptor (continuous line) and the decrease of its intensity due to energy transfer in the presence of HPPC (dashed line).

To determine the displacement constant of $\mathrm{C}_{12} \mathrm{E}_{10}$ by phospholipids on the transmembrane surface of the purified $\mathrm{Ca}^{2+}$ pump $\left(K_{L D}\right)$, the quenching of the intrinsic fluorescence of the pump $\left(I_{d, a} / I_{d}\right)$ by HPPC was determined in the presence of $\mathrm{C}_{12} \mathrm{E}_{10}$ and either soybean phospholipids, membrane phospholipids, or DOPC.

Increasing quantities of $\mathrm{C}_{12} \mathrm{E}_{10}$-HPPC micelles were added to purified $\mathrm{Ca}^{2+}$ pump and mixed until the maxi- 


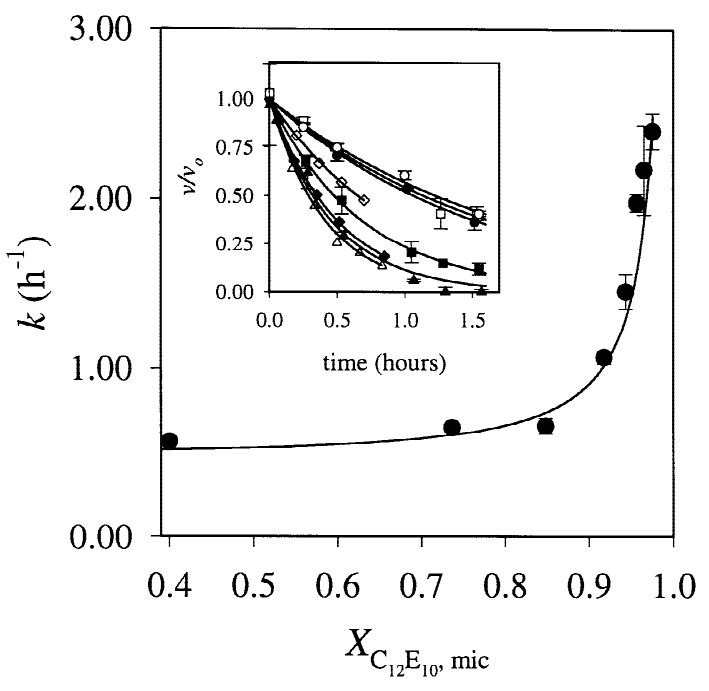

Fig. 3. Dependence of the kinetic coefficient for the thermal inactivation on the $\mathrm{C}_{12} \mathrm{E}_{10}$ mole fraction. $\mathrm{Ca}^{2+}$ pump was purified and reconstituted in storage buffer with $290 \mu \mathrm{M}$ soybean phospholipids and supplemented with $\mathrm{C}_{12} \mathrm{E}_{10}$ up to the following concentrations (mM): 0.8 $(\square), 1.6(\mathbf{\Delta}), 3.2(\diamond), 4.8(\mathbf{\square}), 6.4(\diamond), 8(\mathbf{\Delta})$ and $11.5(\triangle)$. The point at which $\mathrm{C}_{12} \mathrm{E}_{10}$ concentration was $0.2 \mathrm{~mm}(\mathrm{O})$ was achieved by removing the detergent as described in Materials and Methods. Samples were incubated at $44^{\circ} \mathrm{C}, \mathrm{Ca}^{2+}$-ATPase activity was measured as described in Materials and Methods and plotted as a function of the incubation time (inset). Values of $k$ were obtained by fitting Eq. 5 to the experimental data and represented as a function of $X_{D, \text { mic }}$. The best fit of Eq. 10 (continuous line) was achieved with $K_{L D}=10 \pm 2, k_{1}=0.5$ $\pm 0.1 \mathrm{hr}^{-1}$ and $\beta=2$.

mal FRET was observed (about $1 \mathrm{~min}$ ). Then, the calcium pump intrinsic spectrum was registered. Figure $5 A$, $B$ and $C$ shows that $\mathrm{Ca}^{2+}$-ATPase emission intensity diminished with the mole fraction of HPPC. This diminution was enhanced increasing the mole fraction of detergent.

Fluorescence emission spectrum of $\mathrm{Ca}^{2+}$-ATPase in the absence of HPPC was not modified after the addition of detergent over the range indicated in legend of Fig. 5, suggesting that $\mathrm{C}_{12} \mathrm{E}_{10}$ did not cause conformational changes in the protein.

The displacement of $\mathrm{C}_{12} \mathrm{E}_{10}$ in contact with the transmembrane surface of the protein by soybean PL was measured at 25 and $44^{\circ} \mathrm{C}$, and no significant differences were found for $K_{L D}$ values (10 \pm 1 and $9.1 \pm 2.5$, respectively).

\section{Discussion}

In this work, we characterized the thermal stability of purified plasma membrane $\mathrm{Ca}^{2+}$ pump and its dependence on the composition of the micelles where the enzyme is solubilized.

We show that time course of thermal inactivation of this protein is well described by an exponential function.
This is characteristic of first order reactions and indicates that thermal inactivation of $\mathrm{Ca}^{2+}$-ATPase can be kinetically considered as a two-state process involving only fully active and inactive molecules. The temperature dependence of $k$ (Fig. $1 B$ ) is described by a linear Arrhenius plot, suggesting that a common mechanism is responsible for the inactivation between $33-45^{\circ} \mathrm{C}$. This fact allows us to extend the results obtained at a particular temperature to another one within the assayed range. In addition, $E a$ for the thermal inactivation of the pump was in the range of those values found for other membrane and soluble proteins e.g., $300 \mathrm{~kJ} / \mathrm{mol}$ for the sarcoplasmic reticulum $\mathrm{Ca}^{2+}$-ATPase (Davidson \& Berman, 1996) and $205 \mathrm{~kJ} / \mathrm{mol}$ for the RNase (Gekko \& Timasheff, 1981).

The irreversible loss of $\mathrm{Ca}^{2+}$-ATPase activity was not due to either degradation or formation of SDS-stable aggregates of the protein as it was demonstrated by SDSPAGE. Moreover, there is no relation between the inactivation of the $\mathrm{Ca}^{2+}$ pump and the presence of peroxides (polyoxyethyleneglycol detergents may contain peroxides as impurities produced during their synthesis (Møller, le Maire \& Andersen, 1986)). Helenius et al. (1979) reported that addition of 1 mole of BHT per 500 moles $\mathrm{C}_{12} \mathrm{E}_{10}$ is usually enough to prevent peroxides effects. In this work, addition of BHT together with $\mathrm{C}_{12} \mathrm{E}_{10}$ in mole ratios between 1:200 and 1:800 had no effect on $k$.

Throughout this work, we analyzed the effect of the components of the micellar phase on $\mathrm{Ca}^{2+}$-ATPase stability. In this regard, we employed two of the most used phospholipids in the reconstitution of the plasma membrane $\mathrm{Ca}^{2+}$ pump: a pure species (DOPC) and a phospholipid mixture (soybean PL). In addition, we compared the effects of these phospholipids with those of the lipids that constituted the natural environment of the pump.

The influence of the relative concentration of phospholipid and detergent on the thermal stability of the $\mathrm{Ca}^{2+}$-ATPase was studied in two ways: either the concentration of $\mathrm{C}_{12} \mathrm{E}_{10}$ was kept constant while the amount of added soybean PL was varied (Fig. $2 A$ ); or vice versa (Fig. 3). Comparison between these experiments shows that similar values of $k$ were obtained when the mole fractions of the phospholipids in the micelles were similar, even if the phospholipid concentrations in the whole system were different. This indicates that the effect of phospholipids and detergent on the pump stability is related to the micellar phase composition but not to the concentration of micelles in the system.

To interpret this effect, we consider that the $\mathrm{Ca}^{2+}$ pump in the micellar form has its hydrophobic transmembrane surface covered by bound amphiphiles. The existence of a population of lipids with restricted motion corresponding to the first lipid shell surrounding the pro- 

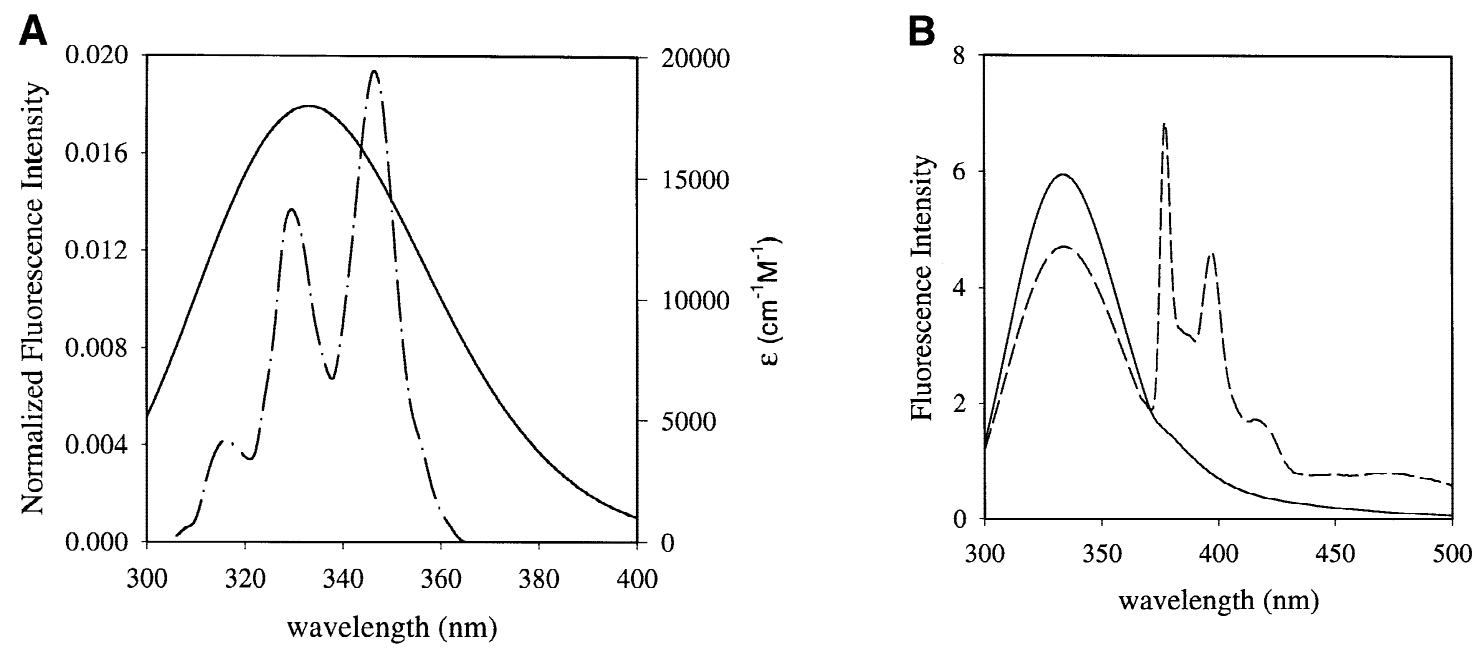

Fig. 4. Resonance energy transfer from $\mathrm{Ca}^{2+}$-ATPase to HPPC. (A) Overlap between the emission spectrum of the Ca ${ }^{2+}$ pump (continuous line) and the absorption spectrum of the HPPC (dashed line). The spectra were determined on the $\mathrm{Ca}^{2+}$-ATPase purified as described in Materials and Methods, and on a micellar preparation of $54.8 \mu \mathrm{M}$ HPPC and $102 \mu \mathrm{M} \mathrm{C}_{12} \mathrm{E}_{10}$. The pyrene-labeled phospholipid concentration in the stock solution was measured changing the solvent by methanol and measuring the absorbance of the resulting solution $\left(\varepsilon=41700 \mathrm{~cm}^{-1} \mathrm{M}^{-1}\right.$ at $\left.341 \mathrm{~nm}\right) .(B)$ Emission spectra of purified $\mathrm{Ca}^{2+}$-ATPase (continuous line) and with the addition of $3.3 \mu \mathrm{M}$ HPPC (dashed lines). HPPC was added in mixed micelles with $\mathrm{C}_{12} \mathrm{E}_{10}$ (mole ratio 0.54). The conditions for the fluorescence measurements are described in Materials and Methods.

tein was previously demonstrated for other integral membrane proteins (for references see Marsh (1997)). The quantitative description of the association of two amphiphiles competing for the hydrophobic transmembrane surface is given by:

$\theta_{L}=\frac{K_{L D} \cdot X_{L, m i c}}{X_{D, m i c}^{\beta}+K_{L D} \cdot X_{L, m i c}}$

where $\theta_{L}$ is the fractional coverage of this surface by phospholipid molecules; $X_{L, m i c}$ and $X_{D, m i c}$ are the mole fractions of lipid and detergent in the micelles, respectively; $K_{L D}$ is the displacement constant of $\mathrm{C}_{12} \mathrm{E}_{10}$ by phospholipids at the hydrophobic surface; and $\beta$ is the exchange number (see Appendix for details). In the case of phospholipid mixtures, $K_{L D}$ is an average coefficient for all the phospholipid molecules interacting with the ATPase (including nonspecific and specific interactions, corresponding to the annular and non-annular sites described by Lee (1998)).

Figures $2 A$ and 3 show that $k$ increases steeply when $X_{D, \text { mic }}$ approaches $1\left(\theta_{L} \rightarrow 0\right)$ and becomes constant at approximately $0.4 \mathrm{hr}^{-1}$ when $X_{L, m i c}$ approaches $1\left(\theta_{L} \rightarrow\right.$ 1 asymptotically). This dependence can be described mathematically by a hyperbolic function of $\theta_{L}$ :

$k=\frac{k_{1}}{\theta_{L}}$

where $k_{1}$ represents the $k$ value for $\theta_{L}=1$. Substituting $\theta_{L}$ with Eq. 8 gives: $k=k_{1} \cdot\left[\frac{X_{D, \text { mic }}^{\beta}}{K_{L D} \cdot X_{L, \text { mic }}}+1\right]$

In this model, detergent and phospholipid molecules are in a dynamic equilibrium competing for the hydrophobic transmembrane sites on the protein; i.e., as the phospholipid concentration in the micellar phase increases, detergent molecules are displaced by lipid molecules. When the immobilized boundary layer is predominantly composed by lipids, the inactivation rate coefficient is minimun $\left(k_{1}\right)$ and therefore, the protein thermal stability is maximal. The continuous lines in Figs. 2 and 3 show that this model agrees with the experimental data.

In addition to the quantitative description of the dependence of the calcium pump thermal stability on the micelle composition, this model provides an indirect determination of the relative affinities of amphiphiles for the hydrophobic transmembrane surface of the protein $\left(K_{L D}\right)$.

To verify the accuracy of the proposed model we developed a FRET method to obtain $K_{L D}$ by a direct measurement of the equilibria between the amphiphiles in contact with the transmembrane surface of the protein and these molecules in the micelles. In this method, a fluorophore-labeled phospholipid (HPPC) compete with the other amphiphiles for the hydrophobic transmembrane domain. The overlap between the emission spectrum of the $\mathrm{Ca}^{2+}$-ATPase and the absorption spectrum of HPPC (Fig. 4A) indicates that these fluorophores constitute an excellent energy transfer donor-acceptor pair. The characteristic Föster distance for the FRET process, 

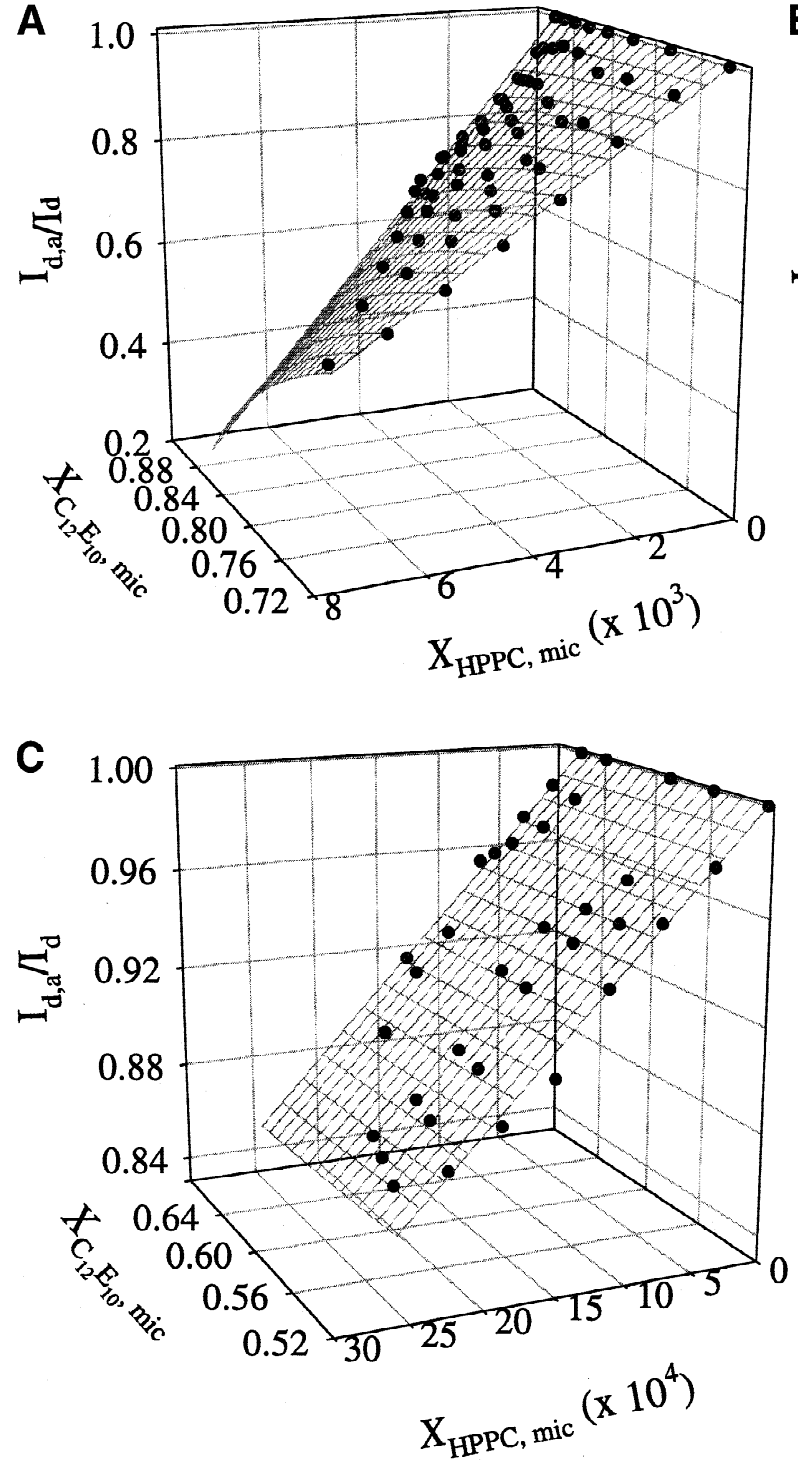

$R_{o}$, was $21.4 \AA$. Considering this value and the dependence of $E$ on the sixth power of the donor-acceptor distance (Lakowicz, 1983), HPPC molecules could only be excited if they were localized in the amphiphile monolayer directly in contact with the hydrophobic transmembrane domain of the $\mathrm{Ca}^{2+}$ pump (see Antollini et al., 1996 and Thomas, Carlsen \& Stryer, 1978 for details of these calculations).

Figure 5 shows that while increasing HPPC mole fraction, the intrinsic calcium pump fluorescence is quenched, indicating that contact sites on the surface of the protein were occupied by HPPC. The energy transfer to the phospholipid probe increases with increasing detergent mole fraction, suggesting that HPPC could replace detergent molecules more easily than phospholipid ones, both in direct contact with the hydrophobic transmembrane domain. As is shown in the legends to Figs.

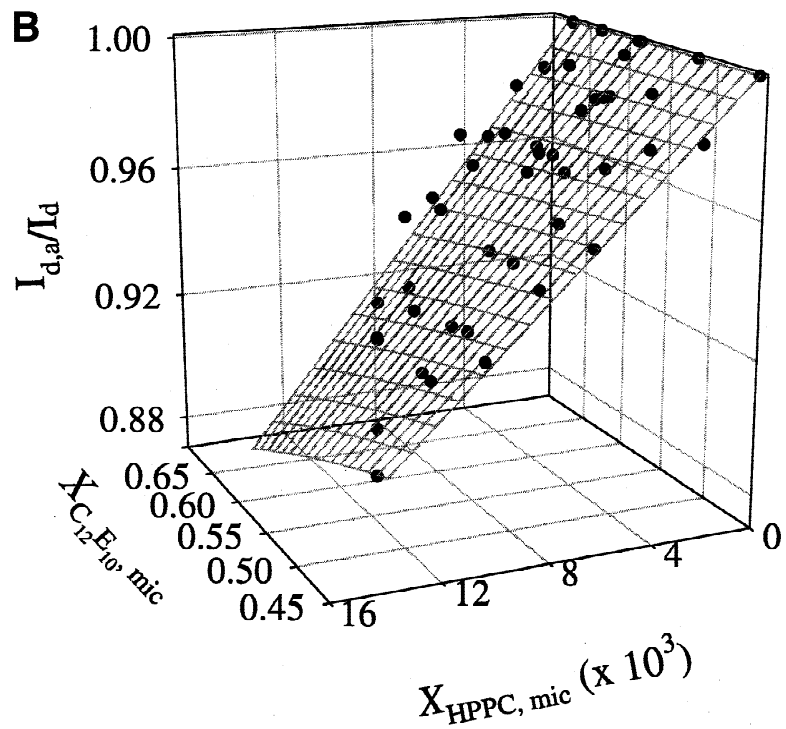

Fig. 5. Dependence of the $\mathrm{Ca}^{2+}$ pump intrinsic fluorescence emission on the mole fractions of HPPC and $\mathrm{C}_{12} \mathrm{E}_{10} \cdot \mathrm{Ca}^{2+}$-ATPase was purified and reconstituted in storage buffer in the prresence of $396 \mu \mathrm{M} \mathrm{C}{ }_{12} \mathrm{E}_{10}$ and $141 \mu \mathrm{M}$ soybean phospholipids $(A), 261 \mu \mathrm{M}$ $\mathrm{C}_{12} \mathrm{E}_{10}$ and $310 \mu \mathrm{M}$ phospholipids extracted from erythrocyte membranes $(B)$ or $824 \mu \mathrm{M} \mathrm{C} \mathrm{C}_{12} \mathrm{E}_{10}$ and $750 \mu \mathrm{M}$ DOPC $(C)$. Samples were supplemented with $\mathrm{C}_{12} \mathrm{E}_{10}$ up to the mole fractions within the ranges: $0.74-0.9(A), 0.46-0.67(B)$ and 0.52-0.6 $(C) . \mathrm{Ca}^{2+}$ pump emission intensity $\left(I_{d, a}\right)$ was measured as indicated in Materials and Methods after adding increasing quantities of HPPC and mixing during $1 \mathrm{~min}$. The final HPPC concentration was always lower than $3.77 \mu \mathrm{M}$. $I_{d}$ values, corresponding to the intensity measured in the absence of HPPC were corrected for the dilution caused by the addition of the probe; this correction was never higher than $7 \%$ of the total intensity. The surface represented in each figure resulted of the fit of Eq. 4 to the experimental data. $K_{L D}$ values that provided the best fit were: $10 \pm 1$ (soybean PL), $3.2 \pm 0.8$ (erythrocyte membranes PL) and $1.4 \pm 0.4$ (DOPC).

2, 3, and 5, $K_{L D}$ values obtained by FRET are not significantly different from those obtained by testing the dependence of the thermal stability of the pump on the micelle composition.

Interestingly, the $K_{L D}$ values measured in micelles of $\mathrm{C}_{12} \mathrm{E}_{10}$ and soybean phospholipid at 25 and $44^{\circ} \mathrm{C}$ were not significantly different. This result indicates that there is not an appreciable dependence of $K_{L D}$ on the temperature in the range $25-44^{\circ} \mathrm{C}$ although the adsorption and desorption rate coefficients for the amphiphiles could change with temperature.

$K_{L D}$ values measured in the three different micelle systems were always greater than 1 indicating that all of the assayed lipids have greater affinity for the protein surface than $\mathrm{C}_{12} \mathrm{E}_{10}$ does. Figure $4 A$ shows that the minimal mole fraction of soybean PL needed to achieve maximal pump stability is nearly 0.15 . In this condition, 
$80 \%$ of the monolayer is composed of PL molecules (calculated applying Eq. 8). In micelles composed of $\mathrm{C}_{12} \mathrm{E}_{10}$ and $\mathrm{DOPC}$ or membrane phospholipids, we verified that this degree of coverage is necessary to attain maximal stability of the $\mathrm{Ca}^{2+}$ pump but, in both cases, $X_{L, \text { mic }} \cong 0.5$ is needed to achieve this value of $\theta_{L}$ (Fig. $4 B$ and $C$, Eq. 8). These results show that the $\mathrm{Ca}^{2+}$ pump requires at least $80 \%$ of the immobilized boundary layer to be composed of lipid molecules to reach maximal thermal stability, regardless of the phospholipid species assayed.

Conversely, the observed differences in $k_{1}$ among the $\mathrm{Ca}^{2+}$-ATPase reconstituted in micelles of different phospholipid composition indicate that the maximal stability of the pump depends on the species of phospholipid used. Among these, soybean PL preserved ATPase activity most effectively; DOPC was the least effective, and an intermediate value of $k_{1}$ was obtained for the erythrocyte PL.

Comparison of $k_{1}$ and $K_{L D}$ among the assayed phospholipids shows that the greater $K_{L D}$, the lower $k_{1}$, suggesting that those phospholipids with higher affinity for the transmembrane surface provide greater stability to the $\mathrm{Ca}^{2+}$ pump. Furthermore, $\mathrm{Ca}^{2+}$-ATPase activity assayed in micelles composed by $\mathrm{C}_{12} \mathrm{E}_{10}$ and different phospholipids (data not shown) increased according to the same sequence that thermal stability and $K_{L D}$ did, suggesting a relation among them.

As suggested by Lee (1998), transmembrane $\alpha$ helices could sense modifications in properties of the bilayer, promoting changes in the conformation of the whole protein and therefore, altering its functional properties. In this way, differences observed in the thermal stability of the pump among the assayed phospholipids (Fig. 4) could be mediated by conformational changes on the transmembrane domain.

It has been shown that acidic phospholipids activate the plasma membrane $\mathrm{Ca}^{2+}$-ATPase by inducing conformational changes (Wrzosek et al., 1989). Analyzing the composition of the assayed phospholipid mixtures, it can be observed that thermal stability increases when the proportion of acidic phospholipids is higher (the percentage of acidic phospholipids is 2-fold higher in soybean PL than in erythrocyte PL). Besides, the addition of phosphatidylserine to the pump reconstituted in DOPC$\mathrm{C}_{12} \mathrm{E}_{10}$ micelles enhanced both the stability and the $K_{L D}$ value (data not shown). The displacement constant for the exchange $\mathrm{C}_{12} \mathrm{E}_{10}$-phosphatidylserine evaluated from these data, according to Eq. A7, indicates that the affinity of phosphatidylserine for the $\mathrm{Ca}^{2+}$ pump transmembrane surface is 6-fold higher than the DOPC one. Therefore, differences on $k_{1}$ and $K_{L D}$ among the assayed phospholipids could be related to the relative abundance of acidic phospholipids in the micelles.

In summary, we have shown that the effect of phos- pholipids and detergent on the $\mathrm{Ca}^{2+}$ pump stability is intimately related to the micellar phase composition. This dependence was modeled assuming that this protein has its hydrophobic transmembrane surface covered by a monolayer of amphiphiles, and that thermal stability depends on the composition of this monolayer. This model explained satisfactory the behavior observed for the thermal stability of the $\mathrm{Ca}^{2+}$-ATPase reconstituted in three different micellar systems. It can be concluded that maximal pump stability is attained when $80 \%$ of the transmembrane protein surface is covered by phospholipid molecules. In addition, the model provides an indirect determination of the relative affinities of amphiphiles for the hydrophobic transmembrane surface of the protein. The agreement of these values with those obtained by using FRET provides a strong evidence to support the proposed model.

We are indebted to Cecilia Arighi for critical reading of the manuscript. This work was supported by CONICET, ANPCYT and UBACYT. V.L. is a fellow of UBA. JPFCR is a career researcher from CONICET. MME is a fellow of CONICET. PRC and LGF are researchers from UBA.

\section{References}

Akaike, H. 1974. A new look at the statistical model identification. IEEE Transactions of Automatic Control 19:716-723

Antollini, S.S., Soto, M.A., Bonini de Romanelli, I., Gutierrez-Merino, C., Sotomayor, P., Barrantes, F.J. 1996. Physical state of bulk and protein-associated lipid in nicotinic acetylcholine receptor-rich membrane studied by laurdan generalized polarization and fluorescence energy transfer. Biophys. J. 70:1275-1284

Atkins, P.W. 1986. Physical Chemistry. W.H. Freeman, New York

Casey, J.R., Reithmeier, R.A. 1993. Detergent interaction with band 3, a model polytopic membrane protein. Biochemistry 32:1172-1179

Castello, P.R., Caride, A.J., González Flecha, F.L., Fernández, H.N., Rossi, J.P., Delfino, J.M. 1994. Identification of transmembrane domains of the red cell calcium pump with a new photoactivable phospholipidic probe. Biochem. Biophys. Res. Commun. 201:194200

Castello, P.R., González Fletcha, F.L., Caride, A.J., Fernández, H.N., Delfino, J.M., Rossi, J.P.F.C. 1997. The membrane topology of the amino-terminal domain of the red cell calcium pump. Protein Sci. 6:1708-1717

Chen, P.S., Toribara, T.Y., Warner, H. 1956. Microdetermination of phosphorus. Anal. Chem. 28:1756-1758

Creighton, T.E. 1993. Proteins. W.H. Freeman and Company, New York

Davidson, G.A., Berman, M.C. 1996. Mechanism of thermal uncoupling of $\mathrm{Ca}^{2+}$-ATPase of sarcoplasmic reticulum as revealed by thapsigargin stabilization. Biochim. Biophys. Acta 1289:187-194

East, J.M., Melville, D., Lee, A.G. 1985. Exchange rates and numbers of annular lipids for the calcium and magnesium ion dependent adenosinetriphosphatase. Biochemistry 24:2615-2623

Fiske, C.H., Subbarow, Y. 1925. The colorimetric determination of phosphorous. J. Biol. Chem. 66:375-400

Förster, Th. 1948. Intermolecular energy migration and fluorescence. Ann. Phys. 2:55-75

Ganong, B.R., Lu, C.M. 1989. Application of nonionic polyoxyethyl- 
ene detergents in studies of phosphatidylinositol kinase. Anal. Biochem. 179:66-71

Gekko, K., Timasheff, S.N. 1981. Mechanism of protein stabilization by glycerol: preferential hydration in glycerol-water mixtures. Biochemistry 20:4667-4676

González Flecha, F.L., Castello, P.R., Gagliardino, J.J., Rossi, J.P. 1999. Molecular characterization of the glycated plasma membrane calcium pump. J. Membrane Biol. 171:25-34

Helenius, A., McCaslin, D.R., Fries, E., Tanford, C. 1979. Properties of detergents. Methods Enzymol. 56:734-749

Lakowicz, J.R. 1983. Principles of Fluorescence Spectroscopy. Plenum Press, New York

Lau, F.W., Bowie, J.U. 1997. A method for assessing the stability of a membrane protein. Biochemistry 36:5884-5892

Lee, A.G. 1998. How lipids interact with an intrinsic membrane protein: the case of the calcium pump. Biochim. Biophys. Acta 1376:381-390

Marsh, D. 1997. Stoichiometry of lipid-protein interaction and integral membrane protein structure. Eur. Biophys. J. 26:203-208

Møller, J.V., Jull, B., le Maire, M. 1996. Structural organization, ion transport, and energy transduction of P-type ATPases. Biochim. Biophys. Acta 1286:1-51

Møller, J.V., le Maire, M, Andersen, J.P. 1986. Uses of non-ionic and bile salt detergents in the study of membrane proteins. In: Progress in Protein-Lipid Interactions. A. Watts and J.J.H.H.M. De Pont, editors. pp. 147-196. Elsevier Biochemical Press, Amsterdam

Neuhoff, V., Arold, N., Taube, D., Ehrhardt, W. 1988. Improved staining of proteins in polyacrylamide gels including isoelectric focusing gels with clear background at nanogram sensitivity using Coomassie Brilliant Blue G-250 and R-250. Electrophoresis 9:255-262

Ostermeier, C., Michel, H. 1997. Crystallization of membrane proteins. Curr. Opin. Struc. Biol. 7:697-701

Popot, J.L., Engelman, D.M. 1990. Membrane protein folding and oligomerization: the two-stage model. Biochemistry 29:4031-4037

Rees, D.C., Komiya, H., Yeates, T.O., Allen, J.P., Feher, G. 1989. The bacterial photosynthetic reaction center as a model for membrane proteins. Annu. Rev. Biochem. 58:607-633

Schägger, H., von Jagow, G. 1987. Tricine-sodium dodecyl sulfatepolyacrylamide gel electrophoresis for the separation of proteins in the range from 1 to $100 \mathrm{kDa}$. Anal. Biochem, 166:368-379

Seber, G.A.F., Wild, C.J. 1989. Nonlinear Regression. John Wiley \& Sons, New York

Silvius, J.R., McMillen, D.A., Saley, N.D., Jost, P.C., Griffith, O.H. 1984. Competition between cholesterol and phosphatidylcholine for the hydrophobic surface of sarcoplasmic reticulum $\mathrm{Ca}^{2+}$ ATPase. Biochemistry 23:538-547

Tanford, C. 1980. The Hydrophobic effect: Formation of micelles and biological membranes. Wiley-Interscience, New York.

Thomas, D.D., Carlsen, W.F., Stryer, L. 1978. Fluorescence energy transfer in the rapid-diffusion limit. Proc. Natl. Acad. Sci. USA 75:5746-5750

Veatch, W., Stryer, L. 1977. The dimeric nature of the gramicidin A transmembrane chanel: conductance and fluorescence energy transfer studies of hybrid channels. J. Mol. Biol. 113:89-102

Volwerk, J.J., Mrsny, R.J., Patapoff, T.W., Jost, P.C., Griffith, O.H. 1987. Multiple equilibria binding treatment of lipid and detergent interactions with membrane proteins. Application to cytochrome c oxidase solubilized in cholate. Biochemistry 26:466-475

Wang, W.Q., Gustafson, A. 1994. Erythrocyte lipid extraction in alcohol-chloroform systems: a comparative study. Acta Chem. Scand. 48:753-758

Wrzosek, A., Famulski, K.S., Lehotsky, J., Pikula, S. 1989. Conformational changes of $\left(\mathrm{Ca}^{2+}-\mathrm{Mg}^{2+}\right)$-ATPase of erythrocyte plasma membrane caused by calmodulin and phosphatidylserine as re- vealed by circular dichroism and fluorescence studies. Biochim. Biophys. Acta 986:263-270

Wu, A., Brand, L. 1994. Resonance Energy Transfer: Methods and Applications. Anal. Biochem. 218:1-13

\section{Appendix}

\section{ADSORPTION ISOTHERM OF AMPHIPHILES TO THE Hydrophobic Transmembrane SuRface of the Plasma Membrane $\mathrm{Ca}^{2+}$ Pump}

We assume that the $\mathrm{Ca}^{2+}$ pump reconstituted in mixed micelles has always its hydrophobic transmembrane surface covered by a shell of amphiphiles whose motion is restricted; this has been demonstrated for many other integral membrane proteins, e.g., sarcoplasmic reticulum $\mathrm{Ca}^{2+}$-ATPase (Silvius et al., 1984). In this context, we consider that (i) adsorption sites on the transmembrane surface can be occupied by any amphiphile, and (ii) the ability of an amphiphile molecule to bind to a specific adsorption site is independent of the occupancy of nearby sites (Atkins, 1986).

We suppose that the surface covered by one phospholipid molecule (contact site) may be occupied by $\beta$ molecules of detergent. According to this assumption, $\beta$ molecules of detergent must be adsorbed or desorbed simultaneously in order to keep the surface covered with amphiphiles when phospholipid-detergent exchange takes place (stoichiometry $1: \beta$ ). The extent of surface coverage by an amphiphile $i$ can be expressed as the fractional coverage $\left(\theta_{i}\right)$ defined as follows:

$\theta_{i}=\frac{\text { number of contact sites occupied by the amphiphile } i}{N}$

where $i$ may be phospholipid, detergent or HPPC and $N$ is the total number of contact sites for phospholipid molecules (e.g., the value of $N$ calculated for the sarcoplasmic reticulum $\mathrm{Ca}^{2+}$-ATPase is in the range 20-30 (Silvius et al., 1984; East, Melville \& Lee, 1985)

The rate of adsorption $\left(v_{a d}\right)$ for each amphiphile is proportional to the probability of a vacancy on the surface $\left(p_{v}\right)$ and to the number of collisions of the molecules with the surface - this last one depending on the mole fraction of the amphiphile in the micelle-then:

$$
\begin{aligned}
v_{a d, L} & =k_{a d, L} \cdot X_{L, \text { mic }} \cdot p_{v} \\
v_{a d, D} & =k_{a d, D} \cdot X_{D, \text { mic }} \beta \cdot p_{v} \\
v_{a d, H P P C} & =k_{a d, H P P C} \cdot X_{H P P C, \text { mic }} \cdot p_{v}
\end{aligned}
$$

where $k_{a d, L}, k_{a d, D}$ and $k_{a d, H P P C}$ are the rate coefficients for the adsorption of phospholipids, detergent and HPPC, respectively. On the other hand, the rate of desorption of each amphiphile is proportional to the number of adsorbed molecules of its corresponding species:

$$
\begin{aligned}
v_{d, L} & =k_{d, L} \cdot \theta_{L} \cdot N \\
v_{d, D} & =\frac{1}{\beta} \cdot k_{d, D} \cdot \theta_{D} \cdot \beta \cdot N \\
v_{d, H P P C} & =k_{d, H P P C} \cdot \theta_{H P P C} \cdot N
\end{aligned}
$$

with $k_{d, L}, k_{d, D}$ and $k_{d, H P P G}$ representing the rate coefficients for the desorption of phospholipids, detergent and HPPC, respectively.

The dynamic equilibrium on the surface implies that the two rates are equal for each amphiphile. When HPPC is not present, these equilibria could be expressed as:

$k_{a d, L} \cdot X_{L, m i c} \cdot p_{v}=k_{d, L} \cdot \theta_{L} \cdot N$ 
$k_{a d, D} \cdot X_{D, m i c}^{\beta} \cdot p_{v}=k_{d, D} \cdot\left(1-\theta_{L}\right) \cdot N$

Dividing Eq. A4 by Eq. A5 and solving for $\theta_{L}$ gives:

$\theta_{L}=\frac{K_{L D} \cdot X_{L, \text { mic }}}{K_{L D} \cdot X_{L, \text { mic }}+X_{D, \text { mic }} \beta} ; K_{L D}=\frac{k_{a d, L} \cdot k_{d, D}}{k_{d, L} \cdot k_{a d, D}}$

where the coefficient $K_{L D}$, which results from the combination of the adsorption and desorption rate coefficients, gives a measure of the relative affinity of the protein surface for the phospholipid molecules with respect to detergent molecules.

$K_{L D}$ for phospholipid mixtures are a linear combination of the exchange constants corresponding to the different species $(j)$ :

$K_{L D}=\frac{\sum_{j} K_{L D_{j}} \cdot X_{L_{j, m i c}}}{X_{L, \text { mic }}}$

Equation A6 is analogous to that proposed by Volwerk et al. (1987) who considered multiple phospholipid-protein and detergent-protein binding equilibria. Those authors suggested a value of 1 or 2 for the coefficient $\beta$.

For the experiments made in the presence of HPPC, the following equilibrium must be considered: $k_{a d, H P P C} \cdot X_{H P P C, m i c} \cdot p_{v}=k_{d, H P P C} \cdot \theta_{H P P C} \cdot N$

Solving Eqs. A5, A6 and A8 for $\theta_{\mathrm{HPPC}}$ gives:

$\theta_{H P P C}=\frac{\frac{k_{a d, H P P C}}{k_{d, H P P C}} \cdot X_{H P P C, \text { mic }}}{\frac{k_{a d, L}}{k_{d, L}} \cdot X_{L, m i c}+\frac{k_{a d, D}}{k_{d, D}} \cdot X_{D, \text { mic }}{ }^{\beta}+\frac{k_{a d, H P P C}}{k_{d, H P P C}} \cdot X_{H P P C, \text { mic }}}$

Considering that most of the contact sites are occupied by unlabeled amphiphiles, the following relation is obtained:

$\theta_{H P P C} \cong \frac{\frac{k_{a d, H P P C}}{k_{d, H P P C}} X_{H P P C, m i c}}{\frac{k_{a d, L}}{k_{d, L}} X_{L, m i c}+\frac{k_{a d, D}}{k_{d, D}} X_{D, m i c} \beta}$

By comparing Eq. A10 with Eq. 4, the following expression for $\xi$ is obtained:

$\xi=\frac{k_{a d, H P P C}}{k_{d, H P P C}} \cdot \frac{k_{d, D}}{k_{a d, D}} \cdot E$ 